\title{
Atom Probe Tomography Investigation on the Effect of Ni Additions on the Site Occupation and Partitioning Behavior in Co-Based Superalloys
}

\author{
Stoichko Antonov ${ }^{1 *}$, Alec Day ${ }^{2,3}$, Hugues Francois Saint $\mathrm{Cyr}^{3}$, Song $\mathrm{Lu}^{1}$, Wendao $\mathrm{Li}^{1}$, and Qiang Feng ${ }^{1}$ \\ 1. Beijing Advanced Innovation Center for Material Genome Engineering, State Key Laboratory for \\ Advanced Metals and Materials, University of Science and Technology Beijing, Beijing, China. \\ 2. Australian Centre for Microscopy and Microanalysis, and School of Aerospace, Mechanical and \\ Mechatronics Engineering, The University of Sydney, Sydney, NSW 2006, Australia. \\ 3. CAMECA Instruments Inc., 5470 Nobel Drive, Madison WI, 53711, USA. \\ * Corresponding author: antonov@ustb.edu.cn;
}

Single crystal Ni-based superalloys have been the material of choice for the hot section turbine blades of jet engines due to their excellent creep and oxidation behavior [1]. However, this class of alloys is reaching its high temperature capability limit, and the demand for improved engine efficiency, requiring higher operating temperatures, is begging for materials that can replace Ni-based superalloys. In 2006, Sato et al. [2] reported a $\gamma^{\prime}$-strengthened Co-Al-W-based alloy, which possesses significantly improved properties over conventional Co-based alloys. Since then, different research groups have studied the effect of alloying additions on the creep behavior and have shown that the creep properties of some CoAl-W-based single crystal alloys are comparable to those of Ni-based superalloys [3]. However, these novel Co-based superalloys still face major application restrictions, e.g., a lower $\gamma^{\prime}$ solvus temperature, microstructural instability at high temperature, and higher alloy density compared to commercial Nibased superalloys. Research focused at improving these shortcomings suggests that CoNi-based alloys are superior with respect to their Ni-free counterparts, as the Ni expands the $\gamma-\gamma^{\prime}$ two-phase field, thereby improving the stability and the temperature capability of the alloys [4]; however, a higher concentration of $\mathrm{Ni}$ alters the $\mathrm{L}_{2}$ formation behavior to be similar to that of Ni-based superalloys. With that respect, only a few studies exist on the preferred site occupancy in the $\mathrm{L} 1_{2}$ lattice of Co-based alloys [5], mainly due to the difficulty of these measurements, although this sort of information is very useful for alloy design as well as behavior modeling. In this study, we compare the elemental partitioning and the site occupation in a $\mathrm{Ni}$-free and a $\mathrm{Ni}$-rich Co-based superalloy, as studied by atom probe tomography (APT).

Single-crystal Co-7Al-8W-4Ti-1Ta in at.\% (Alloy TaTi) and 7 component variant with 30 at.\% Ni and 5 at. \% Cr (Alloy $\mathrm{NiCr}$ ) were subjected to a standard heat treatment of $900^{\circ} \mathrm{C}$ and $1000^{\circ} \mathrm{C}$, respectively, for $50 \mathrm{~h}$ to ensure an equivalent $\gamma^{\prime}$ volume fraction of $\sim 80 \%$. Several APT samples were prepared using an FEI Helios Nanolabs 600 i focused ion beam (FIB). The microtips were polished with $\mathrm{Ga}^{+}$ions down to a $\sim 50 \mathrm{~nm}$ radius using a final energy of $5 \mathrm{kV}$ and a current of $16 \mathrm{pA}$. APT was conducted using a CAMECA LEAP ${ }^{\circledR} 5000 \mathrm{XR}$ and running conditions set as: base temperature of $-243^{\circ} \mathrm{C}(30 \mathrm{~K}), 40 \mathrm{pJ}$ laser pulse energy, pulse repetition rate of $200 \mathrm{kHz}$, and evaporation rate of up to $1.0 \%$. Electron back scattered diffraction (EBSD) was used to measure the exact deviation from the [001] direction of the sample, while post-APT tip image processing was utilized to estimate an evaporation field of $47.9 \mathrm{~V} / \mathrm{nm}$. The data was analyzed using IVAS 3.8.2 software from CAMECA Instruments Inc.

Fig. 1 (a) and (e) show the typical $\gamma-\gamma^{\prime}$ microstructure of Alloy TaTi and NiCr, respectively, while Fig. 1 (b) and (f) show respective 3D reconstructions (where only Co, W, and Ni ions are displayed). Fig. 1 (c) and $(\mathrm{g})$ are magnified views showing the $\{001\}$ atomic planes. Chen et al. [6] used atomic elemental mapping in a HRTEM to show that the Co atoms occupy the A sites in Alloy TaTi, while the Al, W, Ti 
and Ta occupy the B sites. The APT results confirm this behavior, and it should be noted that based on the composition it appears that there would also be some Co anti-sites. A proximity histogram across one $\gamma-\gamma^{\prime}$ interface, Fig 1 (d), confirms the previously reported partitioning, but also shows Co and $\mathrm{Al}$ segregation to the interface. Meanwhile in the $\mathrm{NiCr}$ alloy, the partitioning follows a more classical Nibased alloy behavior, where $\mathrm{Al}$, Ta, and $\mathrm{Ti}$ partition to the $\gamma^{\prime}$ phase, while $\mathrm{Co}, \mathrm{Ni}$, and $\mathrm{Cr}$ partition to the $\gamma$ phase, with the only exception being $\mathrm{W}$ partitioning to the $\gamma^{\prime}$ phase. Some anti-site formation is also required for correct stoichiometry, which would likely be Co atoms, judging by the behavior of Ni-based alloys. In this alloy, $\mathrm{Co}$ and $\mathrm{Ni}$ occupy the A sites, while $\mathrm{Cr}, \mathrm{W}, \mathrm{Al}, \mathrm{Ta}$, and $\mathrm{Ti}$ occupy the B sites, as shown Fig. 1 (g). To illustrate the site occupation of $\mathrm{Ni}$ and $\mathrm{Cr}$ in the $\mathrm{CrNi}$ alloy more quantitatively, a spatial distribution map (SDM) along the $\mathrm{z}$ direction was calculated and the results for select elements are shown in Fig. 1 (h). The Co and Ni show representative peaks at the $\{002\}$ plane locations, i.e., the A sites, while $\mathrm{Cr}$ follows the behavior of $\mathrm{Ti}$ and shows peaks only at the $\{001\}$ plane locations $\sim 0.365$ $\mathrm{nm}$ apart, which is the lattice constant of the $\gamma^{\prime}$ phase. In Ni-based alloys, the occupation of the $\mathrm{Cr}$ atoms is typically in the A sites, although modeling of pure $\mathrm{Ni}_{3} \mathrm{Al}$ suggests that it would occupy the B sites [7]. These results provide a more complete model of the crystal structure of the $\mathrm{L}_{2}$ phase in these new Cobased and CoNi-based superalloys, which can be utilized for different modelling purposes [8].

\section{References:}

[1] RC Reed, “The Superalloys: Fundamentals and Applications", Cambridge University Press, 2006.

[2] J Sato et al., Science 312 (2006), p. 90.

[3] S Lu et al., Metallurgical and Materials Transactions A 49 (2018), p. 4079.

[4] K Shinagawa et al., Materials Transactions 49 (2008), p. 1474.

[5] S Meher and R Banerjee, Intermetallics 49 (2014), p. 138.

[6] Y Chen et al., Scientific Reports 7 (2017), p. 17240.

[7] PAJ Bagot et al., Acta Materialia 125 (2017), p. 156.

[8] The authors acknowledge funding from the Natural National Science Foundation of China [grant No. 51850410518] and the China Postdoctoral Science Foundation [grant No. 2018M630069].
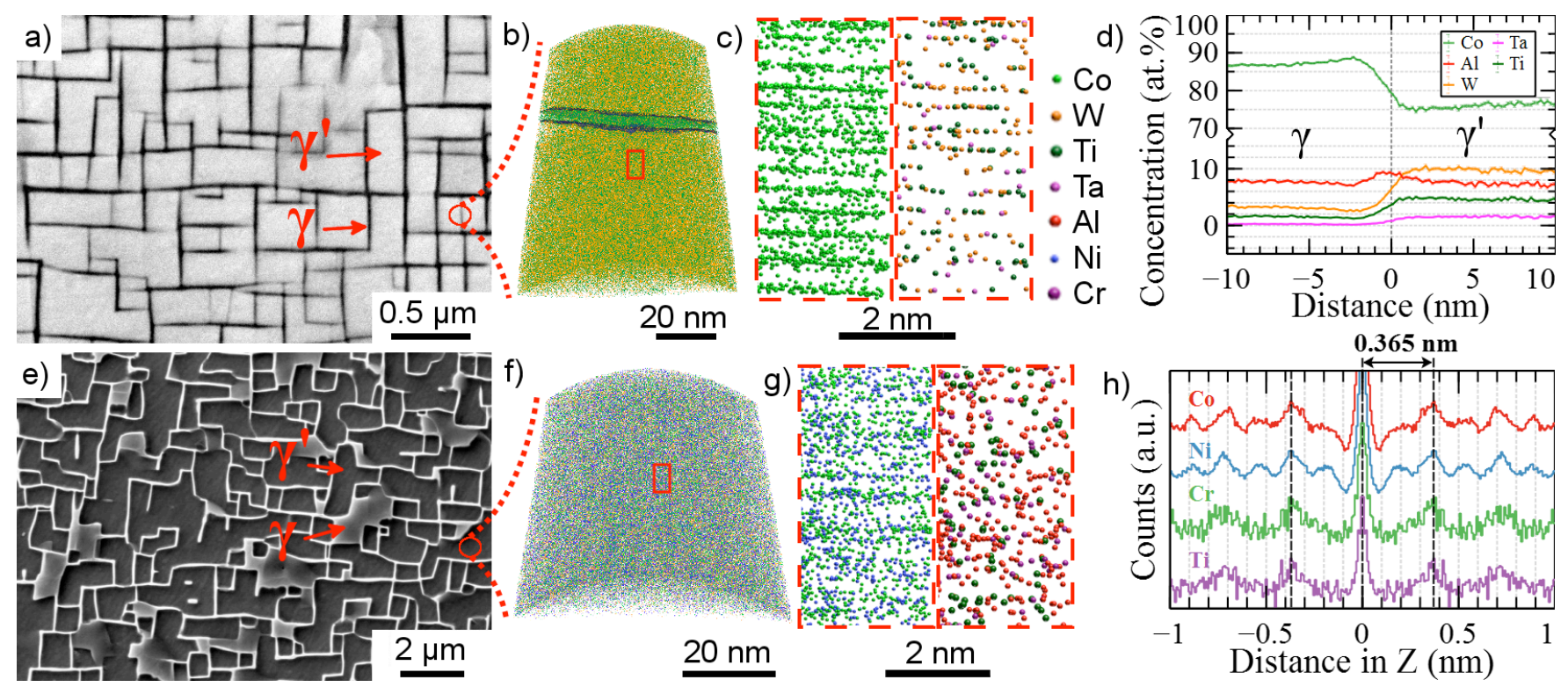

Figure 1. (a) microstructure, (b) $3 \mathrm{D}$ reconstruction, (c) resolved $\{001\}$ atomic planes, and proximity histogram from a 6 at.\% W isoconcentration surface of Alloy TaTi; (e) microstructure, (f) 3D reconstruction, $(\mathrm{g})$ resolved $\{001\}$ atomic planes, and (h) SDM-z of Alloy NiCr. 